\title{
Broadband Radar Cross-Section Reduction Using AMC Technology
}

\author{
Juan Carlos Iriarte, Amagoia Tellechea, José Luis Martínez de Falcón, Iñigo Ederra, Ramón Gonzalo, \\ Member, IEEE, and Peter de Maagt, Fellow Member, IEEE
}

\begin{abstract}
This paper presents the design, fabrication and characterization of a planar broadband chessboard structure to reduce the radar cross-section (RCS) of an object. The chessboard like configuration is formed by combining two artificial magnetic conductor (AMC) cells. The bandwidth limitations intrinsic to AMC structures are overcome in this work by properly selecting the phase slope versus frequency of both AMC structures. 180 degrees phase difference has been obtained over more than $40 \%$ frequency bandwidth with a RCS reduction larger than 10dB. The influence of the incidence angle in the working bandwidth has been performed. A good agreement between simulations and measurements is achieved.
\end{abstract}

Index Terms - Artificial Magnetic Conductor (AMC), Electromagnetic Band Gap (EBG) technology, Radar CrossSection (RCS).

\section{INTRODUCTION}

$\mathrm{B}$ roadband radar cross section (RCS) reduction in a low profile configuration is a challenging objective to designers. At the same time, easy integration with any target which needs to be hidden or with any object for which reflection requires to be minimized is also desired.

Different techniques have been proposed in literature to reduce RCS: shaping, radar absorbing materials, passive or active cancellation. Several solutions have been implemented in each of these RCS reduction techniques [1-10], but narrow band behavior seems to be their common characteristic in low profile designs.

The shaping principle is based on modifying the shape or the surface of the device under test (DUT) in order to redirect the scattered energy away from the source. One way of achieving this scattering goal using a planar configuration was proposed by the authors in [10-11]. The planar structure used, based on a combination of Artificial Magnetic Conductors (AMC) and Perfect Electric Conductors (PEC) in a chessboard

Manuscript received December, 2012. This work was supported by the Spanish Ministry of Economy and Competitiveness Project Nos.TEC-200911995, CSD 2008-00066 and INNPACTO IPT-2011-0960-390000

Juan Carlos Iriarte, Amagoia Tellechea, José Luis Martínez de Falcón, Iñigo Ederra, Ramón Gonzalo are with the Electrical and Electronic Engineer Department, Universidad Pública de Navarra, 31006, Pamplona, Spain (email: ramon@unavarra.es).

Peter de Maagt is with the Antenna and Submillimetre Wave Section, Electromagnetics \& Space Environments Division, European Space Agency (ESA-ESTEC) (e-mail: peter.de.maagt @esa.int) like configuration, has shown the possibility of narrow band RCS reduction. The main advantages of the mentioned structure are its low profile and the possibility to conform it to any object shape while retaining its properties. In addition to this, the structure was simple to manufacture with standard printed board technology.

The working principle of the chessboard configuration is based in the destructive interference between the reflected wave produced by the AMC and PEC cells which compose the chessboard configuration. Metallic cells reflect incident waves with a $180^{\circ}$ phase change while AMC cells introduce a $0^{\circ}$ phase change to the reflected wave at its working frequency. Combining these effects a $180^{\circ}$ phase difference is created between the contribution of the PEC and AMC cells, obtaining destructive interference and achieving a null in the specular direction [10].

The main limitation of the chessboard configuration is the narrow band behavior of the AMC structure. Outside this bandwidth, the AMC behaves as a PEC and the condition for destructive interference is not satisfied anymore. This limitation can be overcome by substituting the PEC cell by another AMC structure operating at a different resonance frequency. This substitution allows the destructive interference condition to be complied with, at least, at the resonance frequency of both AMCs. Consequently, a dual band design can be obtained $[12,13]$.

Apart from the previous results published by the authors in $[12,13]$, other authors have also presented similar structures with the same goals. For instance in $[14,15]$ the combination of two AMCs structures for reducing the RCS is presented. These AMCs structures avoid the use of complex via-hole unit cell configurations achieving frequency bandwidths below $4 \%$. In another work [16], a high permittivity dielectric is used to replace the AMC cells, with the dielectric-AMC and PEC combination acting the same as the one in [12]. However, since the bandwidth of the AMC over which it maintains a zero reflection phase is relatively narrow, it cannot function effectively over a wide frequency band. On the other hand, in [17-18] a method which uses the effective phase differential between two different types of AMCs was developed. In this case the operational bandwidth has been significantly increased, but only normal incidence was studied. As a matter of fact, the performance of the AMC degrades for off-normal incidence. In this paper a novel design, where the AMC 
structures of a chessboard like configuration have been properly selected to control the destructive interference during a larger frequency bandwidth is presented. Preserving the destructive interference in a broadband frequency region results in a broadband RCS reduction structure with low profile, which can easily be integrated with targets. Furthermore, a theoretical analysis of the behavior of the grating lobes as function of the angle of incidence has been included into the paper. This study allows to predict the scattering directions of the Chess-board structure when oblique incidence performance is investigated. These theoretical results have been compared with simulated results.

The paper has been structured as follows; in Section II the theoretical calculation of the grating lobes reflected waves is included, section III focuses on the selection of the AMC configuration, additionally the analysis of its reflection properties is carried out. The predicted scattered radiation and frequency bandwidth results of the overall chessboard structure are shown in Section IV for normal incidence case. Section $\mathrm{V}$ includes the analysis of the same properties for the chessboard configuration but taking into account different angles of incidence. The fabrication process and measured results are included in Section VI, to finalize with the conclusions in section VII.

\section{THEORETICAL CALCULATION OF REFLECTED WAVES DIRECTION}

As proposed in [10], the directions where the reflected power will be redirected can be predicted using conventional array theory. A planar array consisting of counter phase elements $\left(0^{\circ}\right.$ and $180^{\circ}$ phase elements) in a rectangular grid configuration can be used for this prediction. Grating lobes theory fixes the scattering directions. The incident field to the chessboard is scattered to the directions defined by grating lobes supported on the mentioned array. Previous results presented in [10] only include the normal incidence case.

Grating lobes direction for a planar array with elements in phase is shown in [19]. This theoretical result can be adapted to obtain the directions of grating lobes of an array with $180^{\circ}$ progressive phase shift between its constituent adjacent elements. Therefore, the direction of the field reflected by the chessboard configuration can be predicted based on theoretical basics.

Grating lobes appearance direction $\left(\vartheta_{G L}, \phi_{G L}\right)$ for a given chessboard AMC cell dimension $\left(d_{x}, d_{y}\right)$ at a certain frequency can be calculated by complying (1) and (2). The incidence angle is referred as $\left(\vartheta_{0}, \phi_{0}\right)$ and the working wavelength is $\lambda$. $n+m+1$ defines the order of the grating lobes regarding the chessboard structure.

$$
\tan \phi_{G L}=\frac{\sin \vartheta_{0} \sin \phi_{0} \pm(2 n+1) \frac{\lambda}{2 d_{y}}}{\sin \vartheta_{0} \cos \phi_{0} \pm(2 m+1) \frac{\lambda}{2 d_{x}}}
$$

$$
\begin{aligned}
\sin \vartheta_{G L}= & \frac{\sin \vartheta_{0} \sin \phi_{0} \pm(2 n+1) \frac{\lambda}{2 d_{y}}}{\sin \phi_{G L}} \\
= & \frac{\sin \vartheta_{0} \cos \phi_{0} \pm(2 m+1) \frac{\lambda}{2 d_{x}}}{\cos \phi_{G L}}
\end{aligned}
$$

It can be noticed that the theoretical directions of the grating lobes $\left(\vartheta_{G L}, \phi_{G L}\right)$ in an array with counter phase elements are the odd order gratings of the counter phase array configuration. Even gratings are theoretically suppressed by the chessboard structure.

\section{AMC STRUCTURE DESIGN}

A large variety of AMC structures which are appropriate to be used in the proposed chessboard configuration can be found in the literature [20-25]; to mention a few, square patches with and without vias, spirals, Jerusalem crosses, dipoles, rings, etc... After an extensive analysis of their frequency behavior, mainly in terms of the slope of the reflected wave phase curve at the selected frequency region of operation, an AMC structure formed by Jerusalem Crosses (JC) [26, 27] has been selected to create the cells of the chessboard like configuration. No vias have been included to simplify the design and the manufacturing process. As a matter of fact, the vias have no significant effect on the phase slope versus frequency curve [27] and therefore will not affect the AMC performance. ROGERS 3010 material with a thickness of $1.27 \mathrm{~mm}$ and a dielectric constant of 10.2 has been chosen as substrate for the AMC structure. The selected frequency band of operation of the chessboard has been determined to be in the $\mathrm{Ku}$ band.

The two types of cells that constitute the chessboard are the two different arrays of Jerusalem Crosses presented in Fig 1.a. They exhibit a $0^{\circ}$ phase reflection value at different frequencies; $13.5 \mathrm{GHz}$ and $17.9 \mathrm{GHz}$ respectively.

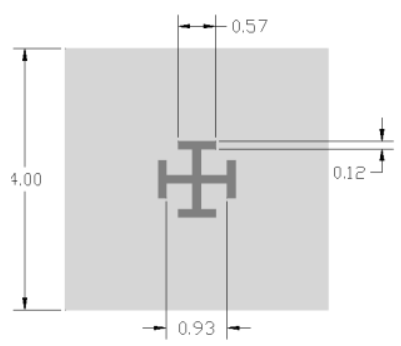

a)

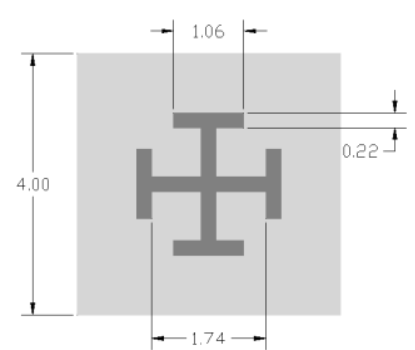

b)
Fig. 1. Jerusalem Cross configuration for the AMC cells (all dimensions in $\mathrm{mm})$.

Each AMC has been independently simulated in AnsysHFSS [28] using a unitary cell with appropriate periodic boundary conditions. This is equivalent to an infinite AMC structure, implemented to obtain the characteristic phase versus frequency curve. Even though the size of the crosses is 
different; the same periodicity has been selected in both AMCs to be able to include an integer number of periods in each type of chessboard cells. Therefore, the final cell length and width will be a multiple of the AMC period.

As aforementioned, the aim of the Chessboard structure is to produce the required destructive interference between the reflected waves from both AMC structures in order to minimize the power reflected in specular direction. Therefore, the dimensions of both Jerusalem Cross AMCs have been optimized to achieve the $180^{\circ}$ difference in the largest possible frequency band. This optimization process results in the dimensions depicted in Fig. 1. The reflection phase versus frequency curves obtained after the optimization process for both AMCs are plotted in Fig. 2.a. The phase difference between the reflections of both AMC structures is shown in Fig. 2.b. The phase difference of the reflected waves stays in the range $180^{\circ} \pm 30^{\circ}$ from $14.4 \mathrm{GHz}$ to $21.8 \mathrm{GHz}(40.88 \%$ bandwidth). Therefore, a broadband behavior (operational band around $41 \%$ ) of the proposed chessboard configuration is expected.

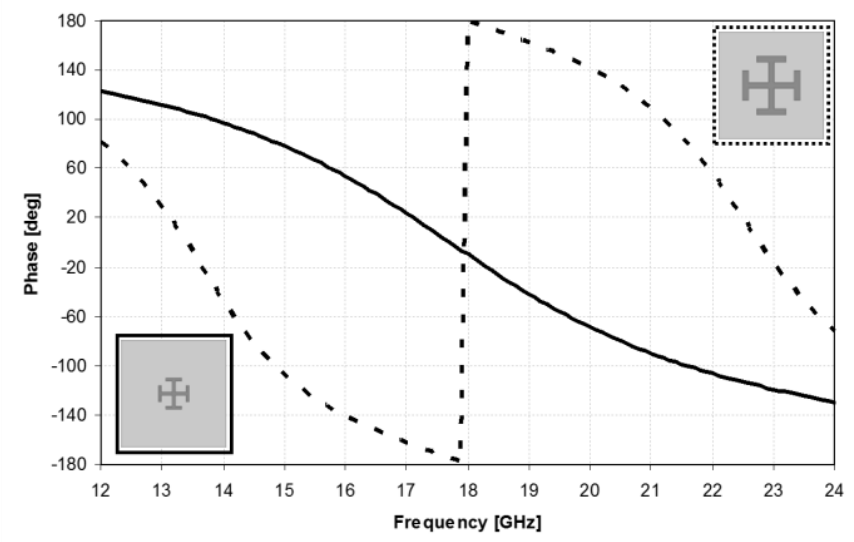

(a)

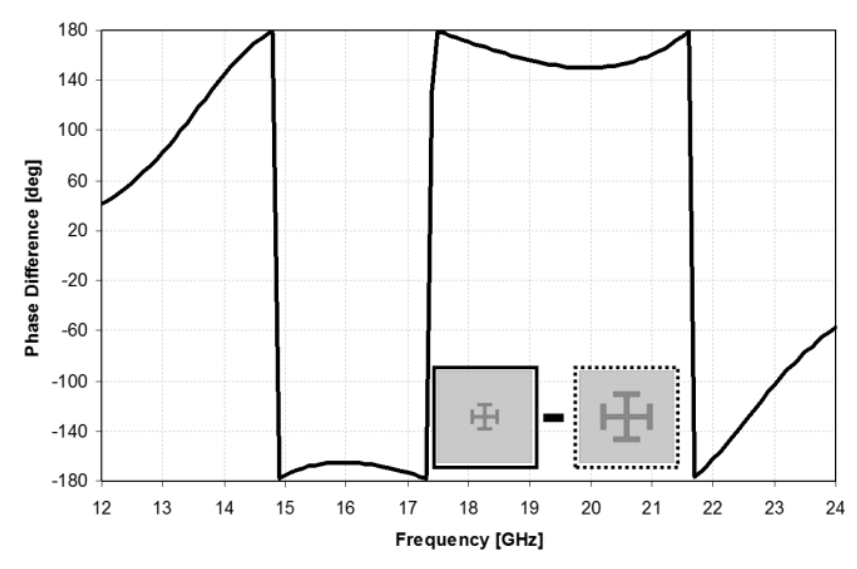

(b)

Fig. 2. (a) Reflection phase variation versus frequency for the two types of Jerusalem Cross AMCs (b) Phase difference versus frequency between both Jerusalem Cross AMCs

\section{BROADBAND CHESSBOARD CONFIGURATION}

Once the AMC structure has been optimized, a unitary $2 \times 2$ chessboard's cell array has been simulated in Ansys-HFSS applying the corresponding boundary conditions to emulate an infinite structure. Each AMC cell is formed by $6 \times 6 \mathrm{JC}$ structures with a total dimension of $24 \times 24 \mathrm{~mm}$ (see Fig. 3). A radiation boundary condition has been defined for the top surface in Fig. 3 to allow the RCS calculation of the structure. This specific AMC cell size has been selected to comply with the distance needed in the diagonal direction of the AMC cell to allow grating lobes appearance in the structure for the lower expected working frequency of the chessboard configuration $(14.4 \mathrm{GHz})$. At the same time, the AMC cell has to be large enough to contain at least four periods of $\mathrm{JC}$ structures to ensure that the AMC behavior $\left(0^{\circ}\right.$ reflection phase) is obtained [10].
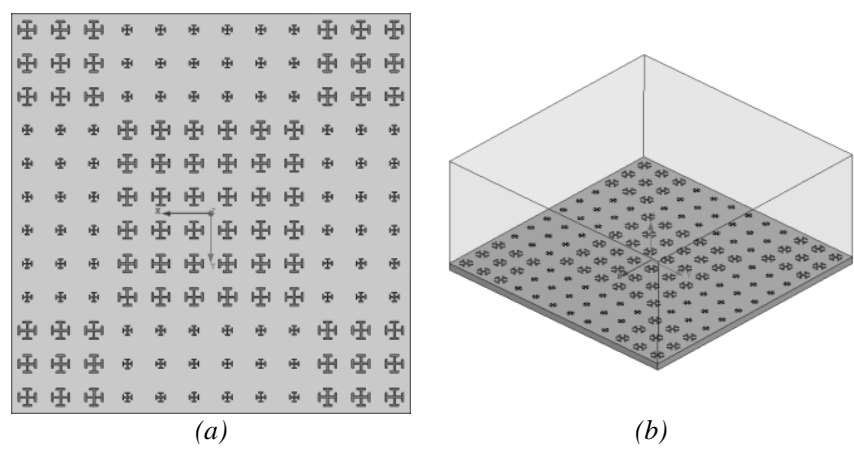

(b)

Fig. 3. Unit cell chessboard structure for the Jerusalem-Cross AMCs (a) Top view (b) Perspective view.

In order to validate the expected broadband characteristic, the monostatic RCS for normal incidence versus frequency has been determined (see Fig. 4). The chessboard RCS value has been normalized with respect to the RCS value of a metallic plate with the same physical dimensions also obtained with Ansys-HFSS. The analysis of Fig. 4 shows an operational bandwidth from $14.5 \mathrm{GHz}$ to $21.8 \mathrm{GHz}(40.22 \%)$ where a reduction of the RCS value larger than $10 \mathrm{~dB}$ is found. The maximum RCS reduction has been obtained at $19 \mathrm{GHz}$ with a value around $-29 \mathrm{~dB}$. The achieved bandwidth is in good agreement with the predicted bandwidth based on the $180^{\circ}$ phase difference between AMCs (Fig. 2.b) calculated in the previous section.

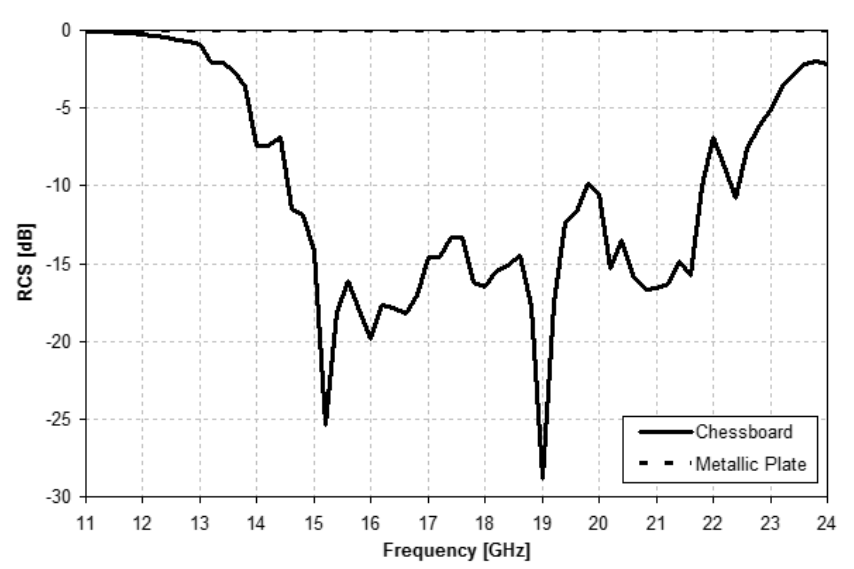

Fig. 4. Normalized monostatic RCS with respect to a metallic plate for the 
proposed broadband chessboard configuration.

The 3-D plot of the scattered field at the frequency where the maximum RCS reduction is achieved $(19 \mathrm{GHz})$ has been depicted in Fig. 5. This plot corresponds to a finite chessboard structure of $10 \times 10$ elements.

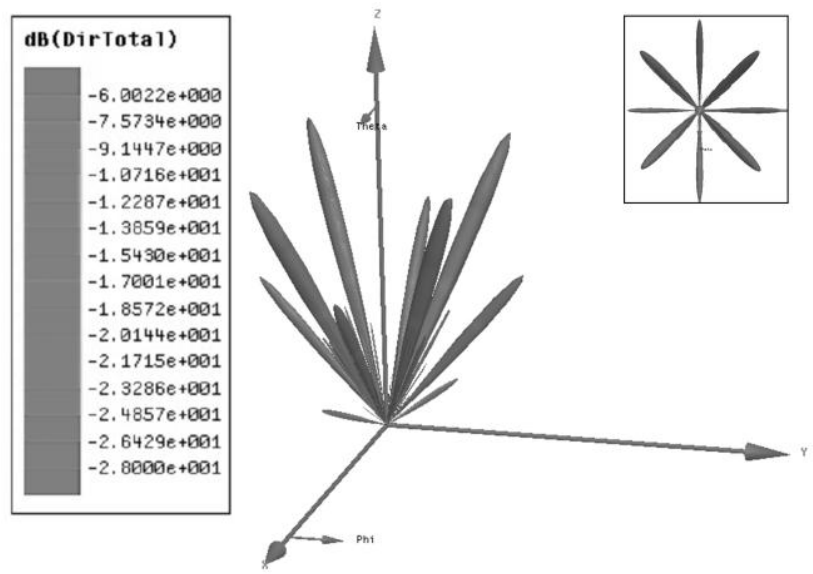

Fig. 5. Scattered field from 10x10 Jerusalem Cross's chessboard structure. The plot is normalized with a metallic plate.

For normal incident field $\left(\vartheta_{0}=0^{\circ}, \phi_{0}=0^{\circ}\right)$ at $19 \mathrm{GHz}$, first order grating lobes $(\mathrm{n}=\mathrm{m}=0)$ are expected based on (1) and (2) at $\pm 28^{\circ}$ for $\pm 45^{\circ}$ phi cuts. Good agreement is shown with the phi $45^{\circ}$ cut of the 3D pattern plotted in Fig. 6.a. The power of the reflected field is about $6 \mathrm{~dB}$ below reference metallic plate for each lobe.

In phi $90^{\circ}$ and phi $0^{\circ}$ cuts the power is redirected to $\vartheta_{G L}= \pm 41^{\circ}$ (see Fig. 6.b). This direction should be canceled by the chessboard configuration according to theoretical results obtained by (1) and (2). In fact, this grating is largely attenuated by the chessboard, being $15 \mathrm{~dB}$ below the amplitude of the reference metallic plate peak. A full cancellation is not achieved because the phase difference does not keep exactly the $180^{\circ}$.

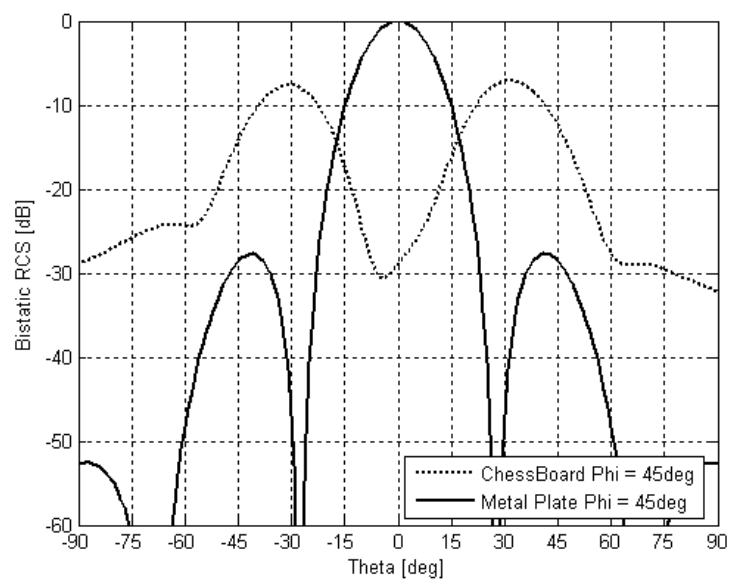

(a)

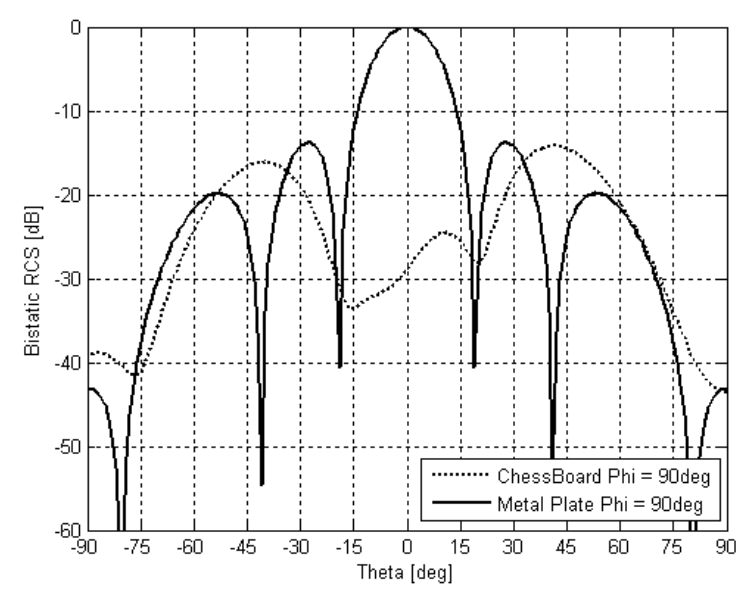

(b)

Fig. 6. Bistatic RCS for the broadband Jerusalem Cross chessboard at 19 $\mathrm{GHz}$ normalized with respect to an equal size metallic plate. Metallic Plate (solid line) and chessboard (dashed line). (a) 45 and (b) 90 phi cuts.

\section{BROADBAND CHESSBOARD INCIDENCE ANGLE VARIATION}

One of the main drawbacks of this chessboard configuration is the dependence of the phase behavior of the AMC structure versus the plane wave incidence angle. The slope of the phase versus frequency curves changes with the incidence angle of the incoming wave. Therefore, the frequency band where $180^{\circ}$ phase difference between curves is obtained varies. As can be seen in Fig. 7, the operational frequency band, where the destructive interference is achieved, is modified as a function of the incident wave angle. In general, the overall bandwidth is reduced when the incidence angle increases.

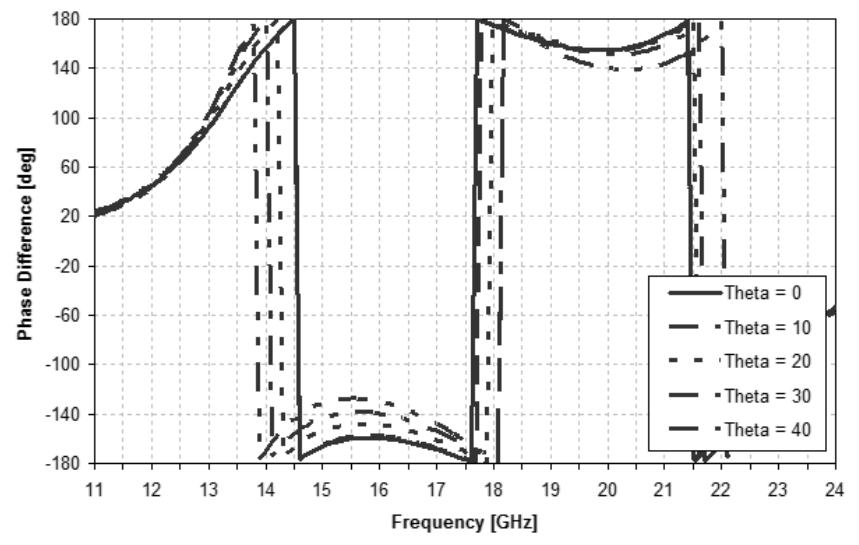

Fig. 7. Phase difference versus frequency between Jerusalem Cross AMCs for several incidence angles.

This is translated into a reduction for the RCS broadband behavior. This phenomenon can be seen in Fig.8. However, the operational bandwidth can be considered as stable for incident angles below $10^{\circ}$. Table 1 summarizes the operational $-10 \mathrm{~dB}$ RCS reduction bandwidth for each incidence angle. 


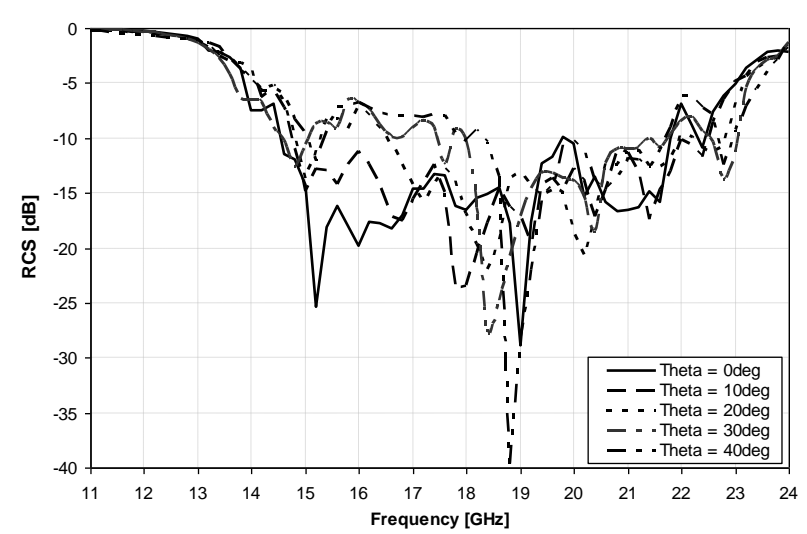

Fig. 8. RCS response of the chessboard configuration for different incident angles of a plane wave normalized with respect to an equal size metallic plate.

\begin{tabular}{|c|c|}
\hline Incident Angle $\left(^{\circ}\right)$ & BW $(\%)$ \\
\hline 0 & 40.2 \\
\hline 10 & 41.1 \\
\hline 20 & 28.6 \\
\hline 30 & 20.0 \\
\hline 40 & 18.4 \\
\hline
\end{tabular}

Table 1. Operational bandwidth values as function of the incidence angle for the proposed chessboard configuration.

The scattered field direction can be predicted by (1) and (2) equations when incident angle is different from normal. As an example of the agreement between theoretical and simulated grating lobes performances, Fig. 9 depicts the 3-D scattered field of the chessboard for a $30^{\circ}$ incident angle at $19 \mathrm{GHz}$.

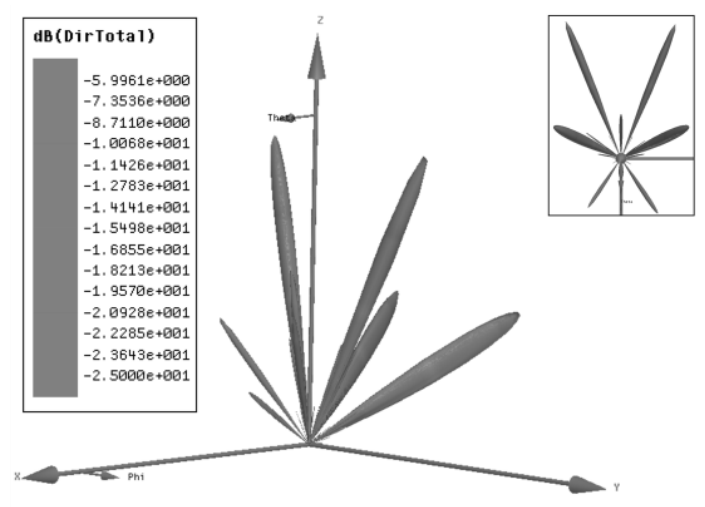

(a)

Fig. 9. 3-D plot of scattered field at $19 \mathrm{GHz}$ when incident angle is $30^{\circ}$.

Applying equations (1) and (2) for an incident angle of $30^{\circ}$, scattered power is theoretically diffracted at $\left(\vartheta_{G L}=-\right.$ $\left.21^{\circ}, \phi_{G L}= \pm 62^{\circ}\right) \quad$ and $\quad\left(\vartheta_{G L}=-63^{\circ}, \phi_{G L}= \pm 21^{\circ}\right)$. The corresponding simulated results (HFSS analysis) are plotted in Fig. 10.a. The grating lobes scattered amplitude values are normalized versus the reflected amplitude of a metallic plate for the same incident angle, resulting $7 \mathrm{~dB}$ and $10 \mathrm{~dB}$ below for each phi cut respectively. Besides, field is also scattered at
$\left(\vartheta_{G L}=35^{\circ}, \phi_{G L}= \pm 34^{\circ}\right)$ with normalized amplitude of $-14 \mathrm{~dB}$ (see the simulated results in Fig. 10.b for comparison proposes).

Good agreement has been obtained between theoretical and simulated results for incidence different from normal. Note that this chessboard configuration for the case of $30^{\circ}$ incident angle not only supports $1^{\text {st }}$ order $(n=m=0)$ grating lobes but also $2^{\text {nd }}$ order GL $(n+m+1=2)$. These last ones have not been included for simplicity.

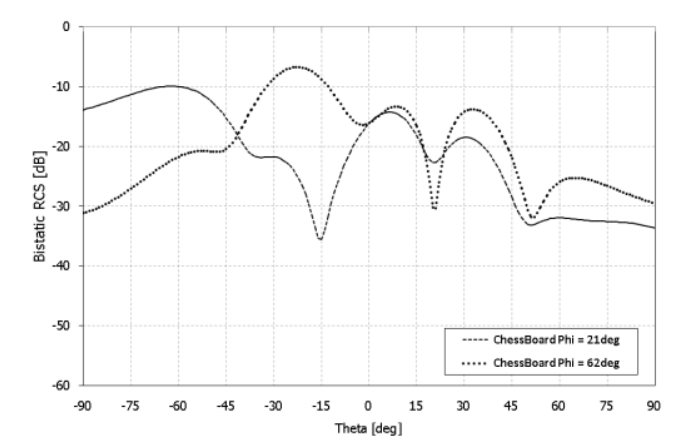

(a)

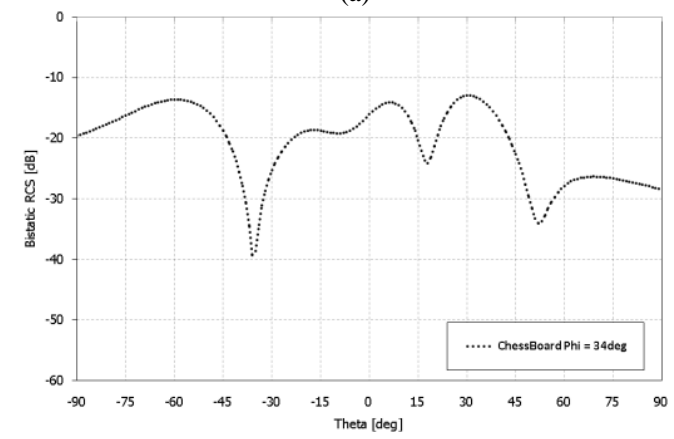

(b)

Fig. 10. 2-D plots of several phi cuts. (a) Cuts of phi $=21^{\circ}$ and $62^{\circ}$, related to $1^{\text {st }}$ order GL of the chessboard structure. (b) Phi $34^{\circ}$ cut, related to $2^{\text {nd }}$ order GL.

\section{FABRICATION AND MEASUREMENTS}

Once the structure has been simulated, a $288 \mathrm{~mm} \times 192 \mathrm{~mm}$ chessboard has been fabricated with a protolaser LPKF 200 machine (a detail of the structure is presented in Fig. 11). This size corresponds with a rectangular structure composed by 18 $\times 12$ unit cells. Each unit cell is at the same time formed by $6 x$ 6 Jerusalem Cross AMC structures respectively and have the dimensions presented in Fig. 1.

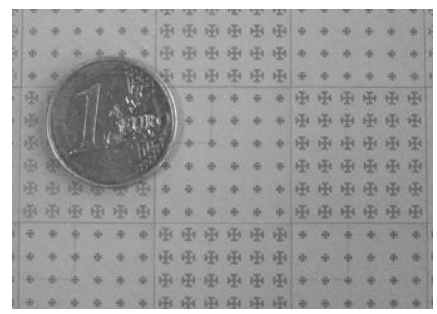

(a)

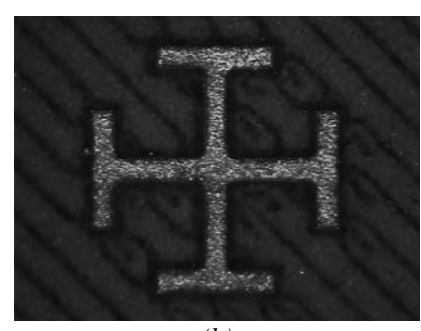

(b)
Fig. 11. (a) Manufactured chessboard structure and (b) detail of the AMC.

A set-up for mono-static and bistatic RCS measurements 
(see Fig. 12) has been used to verify the theoretical results. The chessboard has been mounted in a vertical position, perpendicularly aligned with the emitting antenna. The receiver antenna has been swept in azimuth. Two different pairs of standard gain horn antennas have been used to cover the working frequency band from $12 \mathrm{GHz}$ to $24 \mathrm{GHz}$. These corresponds with the following bands: $11.9 \mathrm{GHz}$ to $18 \mathrm{GHz}$ and $14.5 \mathrm{GHz}$ to $24 \mathrm{GHz}$

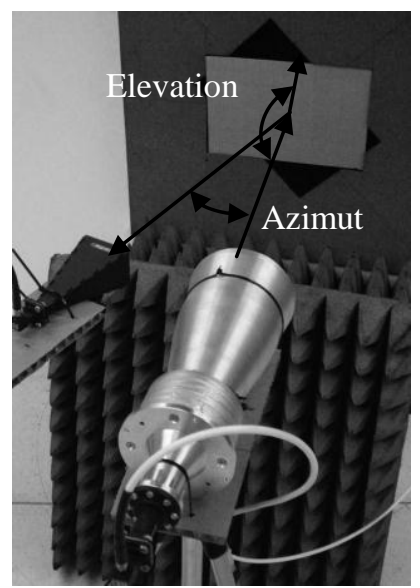

Fig. 12. Measurement Set-up.

The mono-static behavior of the chessboard structure has been evaluated by means of the horn antenna reflection coefficient characterization. The results are depicted in Fig. 13. Note that time domain gating has been applied in order to filter out any undesired reflection; only the reflection coming from the chessboard structure is taken into account. The measured bandwidth is $42 \%$ (from 14.8 to $22.7 \mathrm{GHz}$ ) for the Jerusalem Cross's chessboard configuration. Note that the bandwidth has been calculated with a $-10 \mathrm{~dB}$ reflection coefficient. The measurements are in good agreement with the simulated predictions.

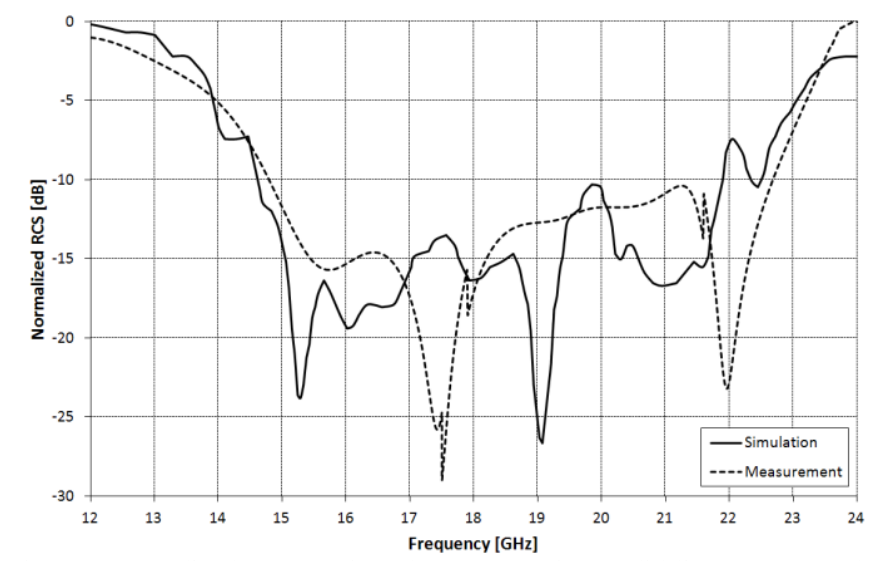

Fig. 13. Normalized monostatic radar cross-section. Solid lines represent the simulated diagram and dashed line the measurements for the Jerusalem Cross broadband chessboard.

The bistatic response, i.e. the scattered field, in the $45^{\circ}$ and $90^{\circ}$ phi cuts have been depicted in Fig. 14 at $17.5 \mathrm{GHz}$ (best value of the measured RCS in Fig. 13). Simulated and measured results are presented either for the reference metallic board as for the proposed chessboard configuration. The scattered power in the different directions is clearly visible. At that frequency, taking into account the chess periodicity, grating lobes are expected based on (1),(2) for normal incidence $\left(\vartheta_{0}\right.$ and $\phi_{0}=0^{\circ}$ ) at $\vartheta_{G L}= \pm 30^{\circ}$ for the $45^{\circ}$ phi cut. Nevertheless, in the $90^{\circ}$ phi cut, attenuated grating lobe can be observed at $\vartheta_{G L}= \pm 41^{\circ}$ for both cases, simulation and measurements due to the loss of the $180^{\circ}$ phase difference between cells.

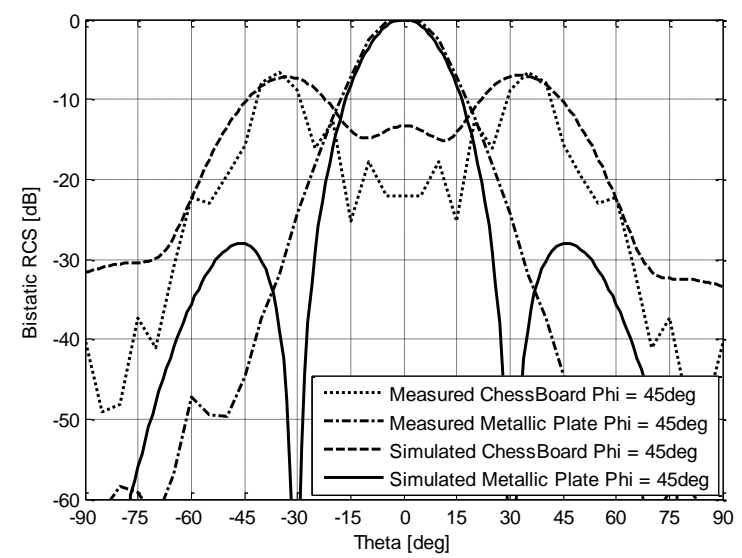

(a)

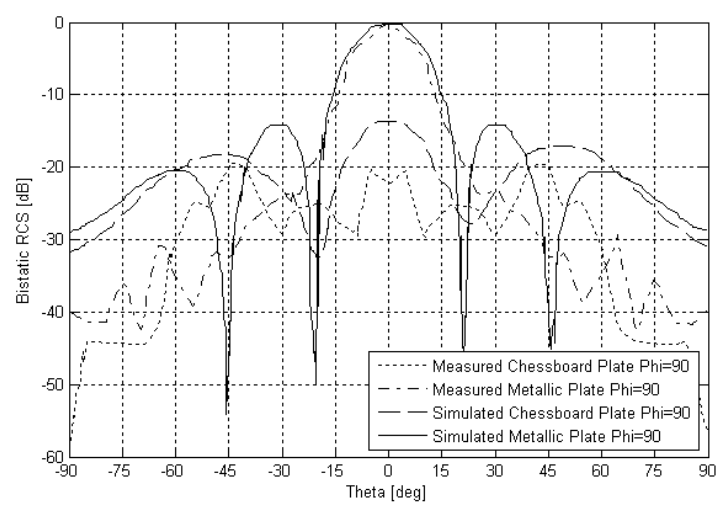

(b)

Fig. 14. Bistatic RCS for the broadband Jerusalem Crosse's chessboard at $17.5 \mathrm{GHz}$ normalized with respect to an equal size metallic plate. Simulated metallic plate (solid line), measured metallic plate (dashdotted line), simulated chessboard (dashed line) and measured chessboard (dotted line). (a) 45 and (b) 90 phi cuts.

Analyzing Fig. 14, the agreement between the theoretical and the measured grating lobes is notable. Furthermore, the grating levels for measurements and simulations at the $45^{\circ}$ cut match well. Nevertheless, the sidelobes for the simulated metallic case are not reproducible in the measurements. It is important to note that although the chessboard structure is placed in the far field of the transmitting horn antenna, some discrepancies are found because the simulations use an ideal incident plane wave.

In order to determinate the functioning of the chessboard configuration; the bistatic scattered field for the $\phi=45^{\circ}$ cut has been measured under normal incidence for the frequency 
range from 12 to $24 \mathrm{GHz}$ (see Fig. 15). For frequencies lower than $14.5 \mathrm{GHz}$ the chessboard configuration behaves as a PEC, i.e. the power is specularly reflected in the same direction as the incident wave. However, when the frequency reaches $14.5 \mathrm{GHz}$, the power is redirected away from the impinging direction. Finally, when the AMC behavior is lost, i.e., the $180^{\circ}$ phase difference, the chessboard configuration behaves again as a conventional PEC. The theoretical angles where the scattered power should be redirected following the grating lobes theory as a function of the frequency are also depicted in Fig. 15 as white lines. It is worth highlighting that at frequencies below $14.5 \mathrm{GHz}$ and above $23 \mathrm{GHz}$ there is no agreement between the white line and the measurements due to the fact that the energy is not scattered in the grating lobe direction because the $180^{\circ}$ phase difference condition is not satisfied between the contributions of both AMC cells. Very good agreement has been obtained between the directions of the reflected power measured in the chessboard structure and the grating lobes direction for frequencies above $14.5 \mathrm{GHz}$. For the negative angle directions shown in Fig. 15 a 5 degrees deviation for the gratings can be seen due to misalignments in the measurement setup.

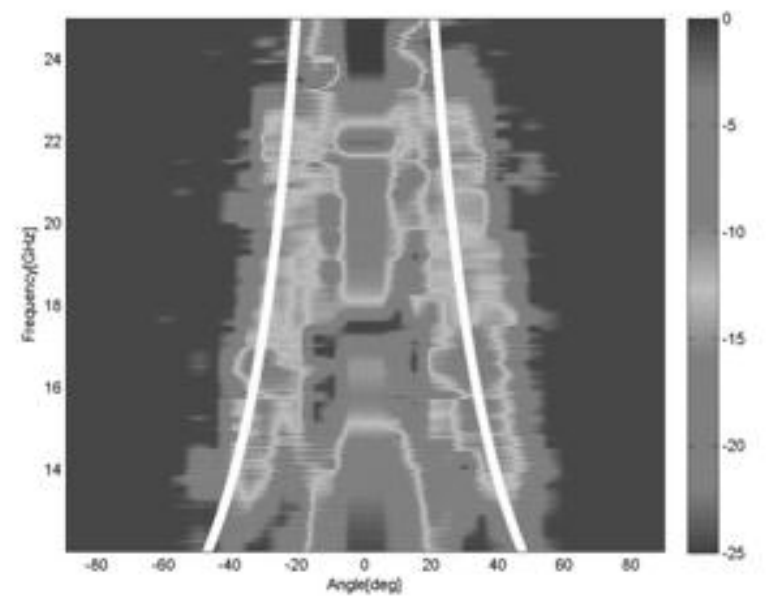

Fig. 15. Measurements of the scattered field under normal plane wave incidence versus frequency. Theoretical grating lobes directions in white.

\section{CONCLUSIONS}

Broadband RCS reduction has been achieved in a planar structure composed by the combination of two AMC structures properly designed to obtain a destructive interference between their reflected waves.

Using AMC structures based on Jerusalem Crosses, the $180^{\circ}$ phase difference between the corresponding reflected phase curves has been obtained over a broad frequency band and for off-normal incident angles. This phase difference creates the destructive interference necessary for the RCS minimization at operational band achieving $40 \%$ working band using a RCS reduction threshold of $-10 \mathrm{~dB}$ with respect to the RCS of a metallic plate. The dependence of bandwidth with the angle of incident wave has been determined either theoretically as simulated. The theoretical results predict adequately the appearance of the grating lobes for directions different from normal ones.

The proposed novel thin structure has been validated both theoretically using Ansys-HFSS as practically by performing measurements with a manufactured prototype. Good agreement has been obtained between simulations and measurements.

\section{REFERENCES}

[1] FanteR. L. and M. T. McCormack, "Reflection properties of the Salisbury screen," IEEE Trans. on Antennas and Propagation, Vol. 36,No. 10, 1443\{1454, Oct. 1988.

[2] Lee, K.-C., Huang, C.-W., and M.-C. Fang, "Radar target recognition by projected features of frequency-diversity RCS", Progress in Electromagnetics Research, Vol. 81, 121-133, 2008.

[3] Li, N.-J., Hu C.-F, Zhang L.-X., and Xu, J.-D., "Overview of RCS extrapolation techniques to aircraft targets", Progress In Electromagnetics Research B, Vol. 9, 249-262, 2008.

[4] Knott E. F., Shaefer J. F., and Tuley M. T., "Radar cross section", 2nd edition, 269-276, Artech House, 1993.

[5] Oraizi H. and Abdolali A., "Combination of MLS, GA \& CG for the reduction of RCS of multilayered cylindrical structures composed of dispersive metamaterials", Progress In Electromagnetics Research B, Vol. 3, 227-253, 2008.

[6] Salisbury, W. W., "Absorbent body for electromagnetic waves", U. S. Patent 2599 944, Jun. 10, 1952.

[7] Abdelaziz A. A., "A novel technique for improving the performance of Salisbury screen", Progress in Electromagnetics Research Letters, Vol. $1,1-8,2008$.

[8] Engheta N., "Thin absorbing screens using metamaterial surfaces", Proc. IEEE Antennas Propagation. Society International. Symposium. 392- 395, 2002.

[9] K. N. Rozanov, "Ultimate thickness to bandwidth ratio of radar absorbers," IEEE Trans. Antennas Propag., vol. 48, no. 8, pp. 12301234, Aug. 2000

[10] Paquay M., Iriarte J. C., Ederra I., Gonzalo R., and de Maagt P., "Thin AMC structure for radar cross-section reduction", IEEE Transactions on Antennas and Propagation, Vol. 55, No. 12, December 2007.

[11] Iriarte, J. C., Paquay M., Ederra I., Gonzalo R., and de Maagt P., "RCS reduction in a chessboard-like structure using AMC cells", Proceedings EUCAP 2007, 1-4, Nov. 11-16, 2007.

[12] Iriarte J. C., Ederra I., Gonzalo R., and de Maagt P., "Dual Band RCS Reduction Using Planar Technology by Combining AMC Structures", Proceedings EuCAP 2009.

[13] Iriarte J.C.; Martinez de Falcon J.L.; Maestrojuan I.; Liberal I.; Rebollo A.; Ederra I.; Gonzalo R.; "Broadband RCS reduction using AMC technology", Proceedings of the 5th European Conference on Antennas and Propagation,1322-1323, 11-15 April 2011

[14] de Cos M. E., Alvarez Y., and Las-Heras F., "A novel approach for RCS reduction using a combination of artificial magnetic conductors", Progress In Electromagnetics Research, Vol. 107, 147-159, 2010.

[15] de Cos, M.E.; Alvarez, Y.; Las-Heras, F.; "RCS reduction using a combination of artificial magnetic conductors", Proceedings of the 5th European Conference on Antennas and Propagation, 1336-1340, 11-15 April 2011

[16] Simms, S., and Fusco, V.: "Chessboard reflector for RCS reduction", Electronic. Letters., 2008, 44, (4), pp. 316-317

[17] Zhang, Y., R. Mittra, B. Z. Wang, and N. T. Huang, "AMCs for ultrathin and broadband RAM design", Electronics Letters, Vol. 45, No. 10, 484-485, 2009.

[18] Zhang, Y., Mittra R., and Wang B. Z., "Novel design for low- RCS screens using a combination of Dual-AMC", Antennas and Propagation Society Intl. Symposium, 2009. APSURSI'09, 1-4, Jun. 1-5, 2009.

[19] Hansen R., "Phased Array Antennas", Wiley Series in Microwave and Optical Engineering, ISBN 0-471-53076-X.

[20] Fu, Y., Li, Y., and Yuan, N., "Wideband composite AMC surfaces for RCS reduction", Microwave and Optical Technology Letters, Vol. 53, No. 4, 715-719, April 2011. 
[21] Akhoondzadeh-Asl L., Hall P., "Comparison of wideband planar dipole antenna performance on EBGs with and without vias", Proceedings of Antennas and Propagation Society International Symposium 2006, IEEE, 2291 - 2294.

[22] Zheng Q.-R., Yan Y.-M., Cao X.-Y., and Yuan N.-C., "High impedance ground plane (HIGP) incorporated with resistance for radar cross section (RCS) reduction of antenna," Progress In Electromagnetics Research, Vol. 84, 307-319, 2008.

[23] Ayad H., Fadlallah M., Youssef H., Elmokdad H., Ndagijimana F., \& Jomaah J. "Performances of low profile dipole antenna AMC-based surface using metamaterials structures". Proceedings of the 2012 19th International Conference on Telecommunications, ICT 2012

[24] Luo Q., Salgado H. M., Moura A. M., \& Pereira, J. R. "Dual-band antenna design using an EBG artificial magnetic conductor ground plane". Proceedings of the 2008 Loughborough Antennas and Propagation Conference, LAPC, 217-220. 2008

[25] Mouffok L., Damaj L., Begaud X., Lepage A. C., \& Diez H. (2011). "Mutual coupling reduction between dual polarized microstrip patch antennas using compact spiral artificial magnetic conductor". Proceedings of the 5th European Conference on Antennas and Propagation, EUCAP 2011, 909-912

[26] Tsao. C. H., and Mittra R., "Spectral-Domain Analysis of Frequency Selective Surfaces Comprised of Periodic Arrays of Cross Dipoles and Jerusalem Crosses", IEEE Transactions on Antennas and Propagation, vol. 32, no. 5, pp. 478-486 (May 1984).

[27] Hosseinipanah M., and Wu Q., "Equivalent Circuit Model for Designing of Jerusalem Cross-Based Artificial Magnetic Conductors", Proceedings of Czech and Slovak Technical Universities, vol. 18, no. 4 (December 2009).

[28] http://www.ansys.com/Products/Simulation+Technology/Electromagneti cs/High-Performance+Electronic+Design/ANSYS+HFSS 